\title{
Hyper-ordered structures and glass-forming abilities of Pd-based metallic glasses
}

\author{
Shinya Hosokawa \\ Institute of Industrial Nanomaterials, Kumamoto University, Kumamoto 860-8555, Japan \\ shhosokawa@kumamoto-u.ac.jp
}

$\mathrm{Pd}_{42.5} \mathrm{Ni}_{7.5} \mathrm{Cu}_{30} \mathrm{P}_{20}(\mathrm{PNCP})$ has at present the most excellent glass-forming ability (GFA) among metallic glasses. The critical cooling rate $(\mathrm{CCR})$ reaches of $0.067 \mathrm{~K} / \mathrm{s}$ and can form a massive bulk glass with a diameter of more than $40 \mathrm{~mm}$ [1]. On the other hand, almost the parent alloys of $\mathrm{Pd}_{40} \mathrm{Ni}_{40} \mathrm{P}_{20}$ (PNP) and $\mathrm{Pd}_{40} \mathrm{Cu}_{40} \mathrm{P}_{20}$ (PCP) have worse CCRs of about $1 \mathrm{~K} / \mathrm{s}$ [2] and $100 \mathrm{~K} / \mathrm{s}$ [3], respectively, indicating that the mixture of $\mathrm{Ni}$ and $\mathrm{Cu}$ elements causes a better $\mathrm{CCR}$ in these bulk metallic glassy alloys.

In order to find a structural origin of the GFAs of these Pd-based glasses, we have carried out anomalous x-ray scattering (AXS) and neutron diffraction (ND) experiments on PNP [4], PCP [5], and PNCP (preliminary results were given in [6]), and the experimental results were analysed by using reverse Monte Carlo (RMC) modelling. The obtained atomic configurations of these alloys were discussed by using a Voronoi tessellation for the short-range atomic arrangements and a persistent homology analysis [7] for the hyper-ordered atomic structures.

Although the general features of the atomic configurations look similar to one another, i.e., most of atomic configurations around all elements are basically icosahedral-type, the main results of these analyses are as follows:

1) A large fraction of "pure" icosahedra are observed around only $\mathrm{Ni}$ atoms (5.8\%) in PNP [4], whereas that around $\mathrm{Cu}$ in PCP is a half value of $2.9 \%$ [5]. Very interestingly in PNCP, large fractions of "pure" icosahedra are detected not only around the Ni atoms of $4.8 \%$, but around the $\mathrm{Cu}$ atoms of $5.6 \%$.

2) Large sizes of partial persistent homology rings are observed for the $\mathrm{Ni} / \mathrm{Cu}$ atoms in all the glasses. However, the size highly depends on the GFA of the glasses, i.e., that in PNCP is slightly larger than in PNP, and much larger than in PCP.

In conclusion, the GFA of Pd-based metallic glasses is not understood as clearly characterized structures such as the existence of clusters of crystal-like fragments. It is realized through hyper-ordered structures, i.e., profound structural features in the short- and intermediate-range atomic order in the glasses.

[1] Nishiyama, N. and Inoue, A., (2002), Appl. Phys. Lett. 80, 568.

[2] Drehman, A. J., Greer, A. L., and Turnbull, D., (1982). Appl. Phys. Lett. 41, 716.

[3] He, Y. and Schwarz, R. B., (1997), Mater. Res. Symp. Proc. 455, 495.

[4] Hosokawa, S. et al., (2019). Phys. Rev. B 100, 054204.

[5] Hosokawa, S. et al., (2021), J. Non-Cryst. Solids 555, 120536.

[6] Hosokawa, S. et al., (2009), Phys. Rev. B 80, 174204.

[7] Hiraoka, T. et al., (2016), Proc. Natl. Acad. Sci. USA 113, 7035.

Keywords: Anomalous x-ray scattering; Neutron diffraction; RMC modelling; Voronoi tessellation; Persistent homology

The author acknowledges Dr. J.-F. Bérar, Dr. N. Boudet, Prof. W.-C. Pilgrim, Prof. L. Pusztai, Dr. S. Hiroi, Dr. S. Kohara, Prof. H. Kato, Dr. H. E. Fischer, and Dr. A. Zeidler for conducting the present investigations. 